\title{
Cognitive Functions of the Cerebellum
}

\author{
Edith V. Sullivan
}

Received: 25 August 2010 /Accepted: 25 August 2010 /Published online: 2 September 2010

(C) Springer Science+Business Media, LLC 2010

The cerebellum, the often forgotten "little brain" at the back the cerebrum, has gained significant attention in the last two decades (Desmond 2010). Although traditionally considered limited in function to component processes of motor control, astute clinicians and researchers posited that such a highly organized brain structure composed of approximately as many neurons as in the cerebrum must have some cognitive function or at least exert some influence on cognitive processes. Despite carefully conducted studies yielding convincing data, papers imputing cognitive function to the cerebellum had been banished to the carousel of rejection. As noted in the paper by Henrietta Leiner, "One peer reviewer actually wrote, 'This couldn't possibly be true or we would have discovered it long ago."” Even current neurology handbooks tend to limit discussion on the outcome of cerebellar pathology to specific motor signs and symptoms.

A change in the course of tradition has required a strong theoretical basis, creative neuroanatomy, perceptive clinical observation, rigorous neuropsychological testing, all complemented by human brain imaging. Forward-thinking scientists in each of these areas contributed to this special issue of Neuropsychology Review on cognitive functions of the cerebellum. The five papers are written from different perspectives on the cerebellum, its far-reaching yet systematic connections with supratentorial brain structures, its functions, and the challenges faced in presenting discoveries inconsistent with traditional beliefs.

\section{E. V. Sullivan $(\bowtie)$}

Department of Psychiatry and Behavioral Sciences,

Stanford University School of Medicine,

401 Quarry Road,

Stanford, CA 94305-5723, USA

e-mail: edie@stanford.edu
Henrietta Leiner, a mathematician, physicist, computer scientist turned neuroscientist, pioneered new horizons in all areas into which she delved. New voices from dogged thinkers formed a critical impetus for rigorous study to challenge cerebellar doctrine about its functions and also its evolutionary purpose. As noted in the current piece, the Leiners (Henrietta and her late husband, Alan) had put forth the proposition that enlargement of the cerebellum in humans, complemented with development of the massive connections with the supratentorium, occurred in step with the emergence of language. Thus, the cerebellum may have enabled rapid language-based thinking and working memory abilities to support speech perception and production.

Jeremy Schmahmann relates his scientific pursuit of cerebellar function through anatomy, neurology, and neuropsychology. His story echoes those of other innovators of new concepts about cerebellar functions, indicating resistance from peers in their ability to break set from the indoctrination of the position that the functions of the cerebellum are solely in the motor domain and cannot contribute to higher-order cognition. Dr. Schmahmann's systematic analysis of the cerebellum's functions has led him to delineate a set of general principles of its functions. Included is what he has identified as the Cerebellar Cognitive Affective Syndrome, which has been a cornerstone for current theories about autism and other neurological disorders with an affective component.

Andreea Bostan and Peter Strick present an anatomicallybased argument that structural interactions between cerebellar and supratentorial structures, including the basal ganglia, provide the communication means for the cerebellum to influence higher-order functions. They review their exquisite tracing studies using rabies virus, which enables identification of multi-synaptic connections traversing extensively across brain systems. The success of these studies has been 


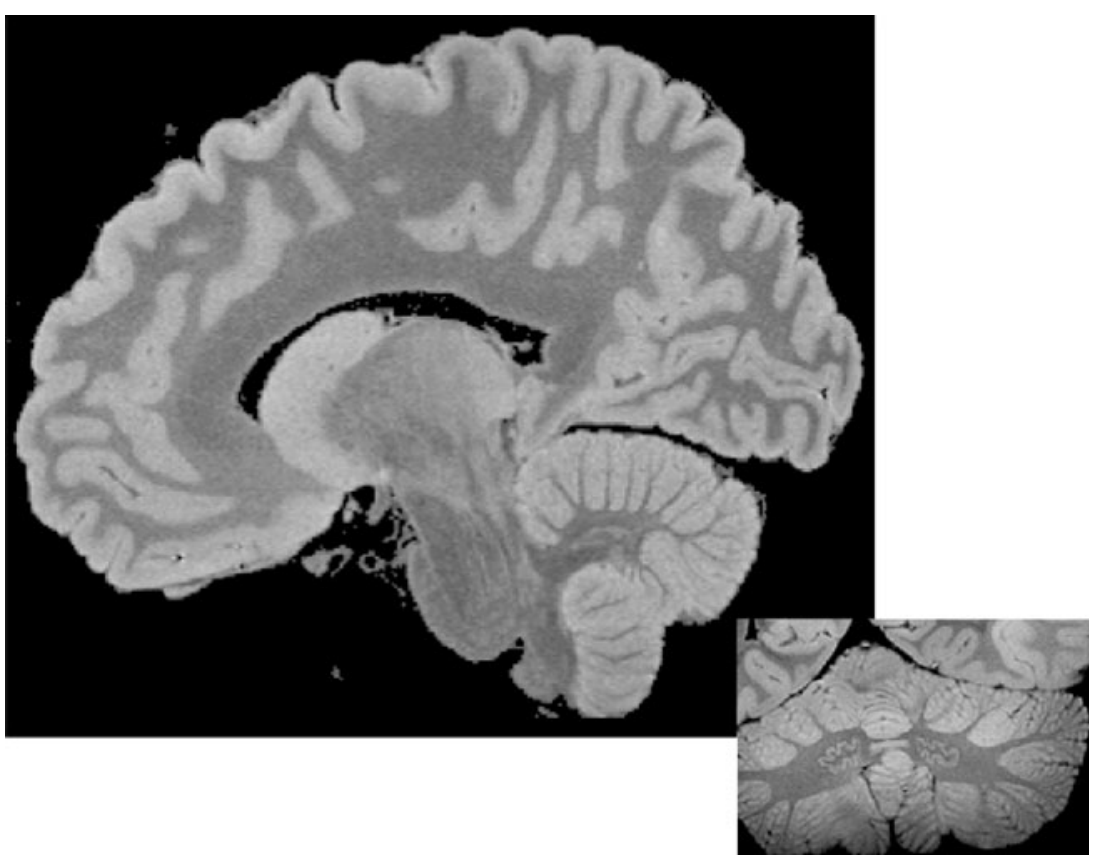

Fig. 1 Cover image. Magnetic resonance images of a formalin-fixed human brain (61 year old woman who died of pulmonary failure). Because of extended image acquisition time $(14 \mathrm{~h})$, this MRI protocol produced images of resolution adequate to reveal typically imperceptible structures, notably in this image, the dentate nucleus of the cerebellum. The large image is a parasagittal slice of the cerebrum and brain stem including the cerebellum; the small inset is a coronal slice of the cerebellum with the dentate nuclei prominently visible

pivotal in providing incontrovertible evidence for a complex system of closed loop bidirectional communication between selective cerebellar and cortical sites and between selective cerebellar and basal ganglial sites in primates.

Cherie Marvel and John Desmond provide replicable support from functional imaging studies on the role of selective regions of the cerebellum in verbal working memory performance in humans. Given the role of the cerebellum as contributing to phonological storage (possibly unique to spoken language) and perhaps even extending working memory capacity, these studies are in keeping with the proposition of the Leiners that the cerebellum, with its great size and high-speed connectivity, with selective regions of the supratentorial brain was essential for human language to evolve.

Natalie Zahr, Anne-Lise Pitel, Sandra Chanraud, and I present a focused review on how human neuroimaging studies of chronic alcoholism have furthered our understanding of cerebellar function. The review includes studies using a variety of magnetic resonance imaging (MRI) methods, including structural MRI, diffusion tensor imaging (DTI), fMRI, and MR spectroscopy in conjunction with bilaterally in the central white matter. Images are modifications from Pfefferbaum, A., Sullivan, E.V., Adalsteinsson, E., Garrick, T., \& Harper, C. (2004). Postmortem MR imaging of formalin-fixed human brain. Neurolmage, 21, 1585-1595 and were produced courtesy of Adolf Pfefferbaum, M.D., Director of the Neuroscience Program, SRI International and Professor Emeritus, Stanford University School of Medicine

neuropsychological study. The central tenet is that disruption of an executive frontocerebellar network is a major contributor to characteristic behaviors of alcoholics that, on the one hand, perpetuate alcohol use disorders possibly through cerebellar conditioning mechanisms, and on the other hand, lead to compensation for frontally-based dysfunctions in alcoholism. As imaging methods improve, we maybe in the position to visualize and quantify the integrity of structures in vivo currently hidden within the cerebellum, such as the dentate nucleus (Fig. 1), in addition to assessing their associated functions.

Acknowledgment This work was supported by grants from the NIAAA (AA010723, AA017168, AA017923) and NIA (AG017919).

Disclosures The author is the editor-in-chief of the journal.

\section{Reference}

Desmond, J. E. (2010). Trends in cerebellar research. Behavioural Neurology, 23, 1-2. 